\title{
Putting nature back into drug discovery
}

\author{
Lutz Müller-Kuhrt
}

The human genome has provided the pharmaceutical industry with innumerable starting points for new drug discovery approaches. And yet, the expected flood of new chemical entities has failed to materialize. What are the reasons for this failure? Could it be that the assay strategy or specific technology used to identify new drug candidates is fundamentally wrong? Is it the complexity of biological systems that represents the insurmountable hurdle? Or are we simply testing the wrong substances? Natural products have declined in popularity since the introduction of synthetic small molecules several years ago by many pharmaceutical companies. But natural products not only complement synthetic molecules, they also exhibit drug-relevant features unsurpassable by any synthetic compound ${ }^{1,2}$.

One key feature of natural products is their enormous structural and chemical diversity ${ }^{3}$. In fact, about $40 \%$ of the chemical scaffolds found in natural products are absent in today's medicinal chemistry ${ }^{4}$, and therefore complementary to synthetically produced molecules. Most possibly this is one of the reasons for their historical success in drug discovery, with $45 \%$ of today's best-selling drugs originating from natural products or their derivatives ${ }^{1}$.

Another important advantage of natural products is that they have 'a biological history.' Biosynthesis of natural products involves repeated interaction with modulating enzymes, and the actual biological function of many natural products comprises binding to other proteins. Thus, the ability of natural products to interact with other molecules, an indispensable prerequisite to making an effective drug, might be considered as biologically validated ${ }^{5}$. It is an unsurprising, but often overlooked, fact that many natural products exhibit advanced binding characteristics compared with synthetics. Most probably, the sterically more complex structure of natural products contributes to this ${ }^{3}$.

Why is the pharmaceutical industry so reluctant to use these substances in their drug discovery programs? One problem is that substances are often rigidly selected on the basis of 'Lipinski's Rule of Five' or related catalogs of

Lutz Mueller-Kuhrt is CEO of AnalytiCon Discovery GmbH, Potsdam, Germany . e-mail: l.mueller-kuhrt@ac-discovery.com parameters defining how a promising synthetic molecule should look ${ }^{6}$. Lipinski himself stated that his 'Rule of Five' is not applicable to natural products. Similarly, optimization procedures may be biased against natural products. Oprea et al. ${ }^{7}$ have analyzed the general differences between leads and drugs to define guidelines for such optimization protocols. Similar to Lipinski, they realized that their findings are obscured by natural products as these molecules fit neither in the 'lead-like' nor in the 'drug-like' space he defined.

Resupply of source material is another problem for both technical and political reasons. Either the raw material is not constantly available in sufficient amounts or political objections claim that biological resources have to be protected. Before the availability of a protocol for chemical synthesis of taxol, its harvesting resulted in the destruction of thousands of Pacific Yew trees.

In addition, as natural products have traditionally only been available as crude extracts, screening such mixtures of substances is a rather uneconomic process because the screening step has to be followed by the crucial work of isolating the active compound from the hit extract. While pure-compound, natural product libraries seem to represent the only practical alternative, they are considered uneconomical, mainly because of high production costs. Moreover, the development of optimization technologies for refining compounds identified in such screens into successful drugs is still in its infancy ${ }^{5}$.

A last challenge in facilitating natural product discovery is to enact appropriate legislation, such as the 'United Nations Convention on Biological Diversity,' to tackle the resource problem, protect the environment and ensure equitable agreements between the countries from which substances are collected and the companies that ultimately commercialize them.

Clearly, many of the above problems arise because natural compounds are treated the same way as synthetically produced small molecules. We apply the same rules, use the same modification technologies and expect the same reactions. Taxol and cyclosporine are illustrative examples of how misleading this approach could be: screening filters currently used in the pharmaceutical industry would have interfered with the detection of these two blockbusters.
Today, high-quality, pure and resuppliable natural products are becoming available. Thus, problems related to extract screening, such as redundancy of compounds, can be eliminated. Furthermore, certain suppliers are now able to deliver fully structure-elucidated, high-purity natural product collections at reasonable prices ${ }^{8}$. Combinatorial libraries based on natural products have also become available ${ }^{2,4}$, enabling promising natural scaffolds to be either produced entirely synthetically by combinatorial chemistry ${ }^{5,9}$ or derivatized with synthetic side chains to increase the 'leadability' of a molecule. As each interesting natural product can be used as origin for a new library, it is likely that several collections of high-potential test molecules will soon be available.

The problems associated with exploiting environmental resources, such as exotic plants and microorganisms, to enlarge the pool of natural-product test substances may also be solved by alternatives to the actual use of raw material taken directly from natural habitats ${ }^{10}$. Indeed, in some cases, it may not be necessary to exploit such sources because $30-50 \%$ of the molecules from nonexotic organisms have not yet been published ${ }^{8}$. Thus, there is still an enormous pool of new substances at our disposal before additional, rare resources need to be explored.

Natural products should no longer be viewed as unapproachable, old-fashioned substances; rather, they should be recognized as accessible, highly variable test molecules. We should embrace the incomprehensibility of nature and the diversity of natural products, and not complain about it. It is exactly this element that we are unable to generate in our laboratories.

\footnotetext{
1. Cragg, G.M., Newman, D.J. \& Snader, K.M. J. Nat. Prod. 60, 52-60 (1997).

2. Tan, D.S., Foley, M.A., Shair, M.D. \& Schreiber, S.L. J. Am. Chem. Soc. 120, 8565-8566 (1998).

3. Henkel, T., Brunne, R.M., Müller, H. \& Reichel, F. Angew. Chem. Int. Ed. 38, 643-647 (1999).

4. Lee, M.-L. \& Schneider, G.J. Comb. Chem. 3, 284-289 (2001).

5. Brohm, D. et al. Angew. Chem. Int. Ed. 2, 307-311 (2002)

6. Lipinski, C.A., Lombardo, F., Dominy, B.W. \& Feeney, P.J. Adv. Drug Deliv. Rev. 23, 3-25 (1997).

7. Oprea, T.I., Davis, A.M., Steague, S.J. \& Leeson, P.D. J. Chem. Inf. Comput. Sci. 41, 1308-1315 (2001).

8. Bindseil, K.U. et al. Drug Disc. Today 6, 840-847 (2001).

9. Nicolaou, K.C. et al. Nature 387, 268-272 (1997).
}

10. Harvey, A. Drug Disc. Today 5, 294-300 (2000). 